\title{
Advances in Olericulture
}

\section{Series editor}

Silvana Nicola, Department of Agricultural, Forest and Food Sciences, DISAFA, Vegetable Crops \& Medicinal and Aromatic Plants, VEGMAP, University of Turin, Grugliasco (Turin), Italy 
The book series Advances in Olericulture provides a state-of-the-art account of research in olericulture, the applied life science of production and utilization of vegetable crops. The series focuses on various aspects of vegetable science and technology covering primarily but not exclusively species where the vegetative organ is the economically important component. The series of books spans current topics from sustainable fertilization to organic production; from open field cultivation to advanced soilless growing techniques; from vegetable seed and seedling physiology to vegetable quality and safety; from environmental stresses to phyllosphere communities interaction with vegetables; from postharvest biology and technology to minimally processing of vegetables. The series is designed to present the most advanced scientific information available linking basic and applied research for serving olericulturists, research workers, teachers and advanced students.

More information about this series at http://www.springer.com/series/11965 
Francesco Tei • Silvana Nicola - Paolo Benincasa Editors

\section{Advances in Research on Fertilization Management of Vegetable Crops}

囪 Springer 


\section{Editors}

Francesco Tei

Department of Agricultural,

Food and Environmental Sciences

University of Perugia

Perugia, Italy

\author{
Paolo Benincasa \\ Department of Agricultural, \\ Food and Environmental Sciences \\ University of Perugia \\ Perugia, Italy
}

\author{
Silvana Nicola \\ Department of Agricultural, Forest and Food \\ Sciences, DISAFA, Vegetable Crops \& \\ Medicinal and Aromatic Plants, VEGMAP \\ University of Turin \\ Grugliasco (Turin), Italy
}

ISSN 2367-4083

Advances in Olericulture ISBN 978-3-319-53624-8 DOI $10.1007 / 978-3-319-53626-2$
ISSN 2367-4091 (electronic)

$$
\text { ISBN 978-3-319-53626-2 (eBook) }
$$

\section{Library of Congress Control Number: 2017938795}

(C) Springer International Publishing AG 2017

This work is subject to copyright. All rights are reserved by the Publisher, whether the whole or part of the material is concerned, specifically the rights of translation, reprinting, reuse of illustrations, recitation, broadcasting, reproduction on microfilms or in any other physical way, and transmission or information storage and retrieval, electronic adaptation, computer software, or by similar or dissimilar methodology now known or hereafter developed.

The use of general descriptive names, registered names, trademarks, service marks, etc. in this publication does not imply, even in the absence of a specific statement, that such names are exempt from the relevant protective laws and regulations and therefore free for general use.

The publisher, the authors and the editors are safe to assume that the advice and information in this book are believed to be true and accurate at the date of publication. Neither the publisher nor the authors or the editors give a warranty, express or implied, with respect to the material contained herein or for any errors or omissions that may have been made. The publisher remains neutral with regard to jurisdictional claims in published maps and institutional affiliations.

Printed on acid-free paper

This Springer imprint is published by Springer Nature

The registered company is Springer International Publishing AG

The registered company address is: Gewerbestrasse 11, 6330 Cham, Switzerland 


\section{Preface}

In the last decades, research on fertilization management in vegetables aimed at producing economical yields with reduced fertilizer inputs by the development and implementation of cropping systems, nutrient management approaches and crop varieties. Examples of the interventions in cropping systems include adequate crop rotations, intercropping, double cropping and other strategies for a better soil organic matter management; nutrient management approaches include modelling, decision support systems, crop nutritional status testing and precision agriculture technologies; amelioration of crop varieties has been directed towards higher nutrient/fertilizer use efficiency. Hence, the aim of this book is to review the recent literature on the key scientific and technical subjects of fertilization management in vegetable crops.

The book consists of ten chapters.

Chapter "The Role of Research for a Sustainable Fertilization Management in Vegetables: Future Trends and Goals", by the editors of the book, is the introduction to the book, presenting the importance of the fertilization as one of the agricultural practices in vegetable production and the rationale of the need for enhancing efficient fertilization strategies for the twenty-first century.

Chapter "Tools and Strategies for Sustainable Nitrogen Fertilisation of Vegeta ble Crops", by Thompson et al., presents and discusses the tools and strategies for sustainable nitrogen fertilization, including methods for soil analysis or estimation of the soil $\mathrm{N}$ supply, $\mathrm{N}$ balance calculations, methods based on plant analysis, methods based on monitoring crops with optical sensors and the use of computerized decision support systems based on simulation models.

Chapter "Organic Matter Mineralization as a Source of Nitrogen", by De Neve, is focused on the organic matter mineralization as a source of nitrogen. It provides details on the biotic and abiotic factors governing the process, introducing simple empirical equations that allow making rapid estimates of $\mathrm{N}$ mineralization, describing the different types of organic materials with respect to expected $\mathrm{N}$ availability and pointing out the importance of synchronizing $\mathrm{N}$ mineralization with crop $\mathrm{N}$ demand. 
Chapter "Fertilizers: Criteria of Choice for Vegetable Crops", by Sambo and Nicoletto, reviews the main mineral fertilizers and traditional and innovative organic materials (i.e. compost, sewage sludge, anaerobic digestion residues and spent mushrooms compost) and the criteria of choice for vegetable crops.

Chapter "Crop Rotation as a System Approach for Soil Fertility Management in Vegetables", by Benincasa et al., deals with crop rotation as one of the key strategies of conservative agriculture, aimed at guaranteeing the long-term productivity and sustainability of vegetable cropping systems. Mineral and organic fertilization, crop residue management, cover cropping and green manuring and intercropping are examined in the frame of crop rotations in conventional and organic systems for either specialized or non-specialized vegetable production.

Chapter "Localized Application of Fertilizers in Vegetable Crop Production", by Simonne et al., focuses on principles and practices of the localized application (i.e. modified broadcast method, banding application method, fertigation method) in vegetable crop production in order to increase the uptake rate of applied nutrients, thereby reducing the application rates, the fertilization cost and the environmental impact of vegetable production.

Chapter "Water and Nutrient Supply in Horticultural Crops Grown in Soilless Culture: Resource Efficiency in Dynamic and Intensive Systems”, by Pignata et al., analyses fertilization management for the different soilless culture systems for efficient and effective control of product quality and environmental sustainability in vegetable crop production. The chapter presents the characteristics and the controls of the substrate-based and liquid-based soilless culture systems in relation to irrigation and fertigation applications, both in open-cycle and closed-cycle hydroponic systems.

Chapter "Plant Breeding for Improving Nutrient Uptake and Utilization Effi ciency”, by Ferrante et al., deals with physiological, biochemical and molecular traits affecting nitrogen uptake by roots and new plant breeding approaches for improving nutrient uptake and utilization efficiency in plants.

Chapter "Water Management for Enhancing Crop Nutrient Use Efficiency and Reducing Losses", by Gabriel and Quemada, covers water management strategies oriented towards improving nutrient use efficiency, reducing nutrient losses and maintaining farm profitability in horticultural systems.

Chapter "An Economic Analysis of the Efficiency and Sustainability of Fertili zation Programmes at the Level of Operational Systems, with Case Studies on Table Tomato, Carrot and Potato in Central Italy", by Martino et al., presents an economic analysis of the efficiency and sustainability of fertilization programmes conducted at the farm level and framed into a conceptualization of the relationship between the decisional and operational systems.

Perugia, Italy

Francesco Tei

Grugliasco (Turin), Italy

Paolo Benincasa

Perugia, Italy

Silvana Nicola 


\section{Contents}

The Role of Research for a Sustainable Fertilization Management in Vegetables: Future Trends and Goals . . . . . . . . . . . . . . 1

Francesco Tei, Silvana Nicola, and Paolo Benincasa

Tools and Strategies for Sustainable Nitrogen Fertilisation of Vegetable Crops . . . . . . . . . . . . . . . . . . . . . . 1

Rodney B. Thompson, Nicolas Tremblay, Matthias Fink, Marisa Gallardo, and Francisco M. Padilla

Organic Matter Mineralization as a Source of Nitrogen . . . . . . . . . 65 Stefaan De Neve

Fertilizers: Criteria of Choice for Vegetable Crops . . . . . . . . . . . 85

Paolo Sambo and Carlo Nicoletto

Crop Rotation as a System Approach for Soil Fertility Management in Vegetables . . . . . . . . . . . . . . . . . . . . . . . . . 115

Paolo Benincasa, Giacomo Tosti, Marcello Guiducci, Michela Farneselli, and Francesco Tei

Localized Application of Fertilizers in Vegetable Crop Production . . . . 149 Eric H. Simonne, Aparna Gazula, Monica Ozores-Hampton, Jim DeValerio, and Robert C. Hochmuth

Water and Nutrient Supply in Horticultural Crops Grown in Soilless Culture: Resource Efficiency in Dynamic and Intensive Systems . . . . . 183 Giuseppe Pignata, Manuela Casale, and Silvana Nicola

Plant Breeding for Improving Nutrient Uptake and Utilization

Antonio Ferrante, Fabio Francesco Nocito, Silvia Morgutti, and Gian Attilio Sacchi 
Water Management for Enhancing Crop Nutrient Use Efficiency and Reducing Losses . . . . . . . . . . . . . . . . . . . . . . . . . . . . . . . . 247 Jose L. Gabriel and Miguel Quemada

An Economic Analysis of the Efficiency and Sustainability of Fertilization Programmes at the Level of Operational Systems, with Case Studies on Table Tomato, Carrot and Potato in Central Italy . . . . . . . . . . . . . . . . . . . . . . . . . . . . . . . 267

Gaetano Martino, Paolo Polinori, and Luca Turchetti

Index . . . . . . . . . . . . . . . . . . . . . . . . . . . . . . . 299 


\section{Contributors}

Paolo Benincasa Department of Agricultural, Food and Environmental Sciences, University of Perugia, Perugia, Italy

Manuela Casale Department of Agricultural, Forest and Food Sciences, DISAFA, Vegetable Crops \& Medicinal and Aromatic Plants, VEGMAP, University of Turin, Grugliasco (Turin), Italy

Stefaan De Neve Department of Soil Management, University of Ghent, Ghent, Belgium

Jim DeValerio Institute of Food and Agricultural Sciences, Florida Cooperative Extension Service, University of Florida, Gainesville, FL, USA

Michela Farneselli Department of Agricultural, Food and Environmental Sciences, University of Perugia, Perugia, Italy

Antonio Ferrante Department of Agricultural and Environmental Sciences Production, Landscape, Agroenergy, Università degli Studi di Milano, Milan, Italy

Matthias Fink IGZ - Leibniz Institute of Vegetable and Ornamental Crops, Großbeeren, Germany

Jose L. Gabriel Department of Agricultural Production and CEIGRAM, Technical University of Madrid, Madrid, Spain

Marisa Gallardo Department of Agronomy, University of Almeria, Almeria, Spain

Aparna Gazula Institute of Food and Agricultural Sciences, Florida Cooperative Extension Service, University of Florida, Gainesville, FL, USA

Current address: Santa Clara County, University of California Cooperative Extension, San Jose, CA, USA 
Marcello Guiducci Department of Agricultural, Food and Environmental Sciences, University of Perugia, Perugia, Italy

Robert C. Hochmuth Institute of Food and Agricultural Sciences, Florida Cooperative Extension Service, University of Florida, Gainesville, FL, USA

Gaetano Martino Department of Agricultural, Food and Environmental Sciences, University of Perugia, Perugia, Italy

Silvia Morgutti Department of Agricultural and Environmental Sciences Production, Landscape, Agroenergy, Università degli Studi di Milano, Milan, Italy

Silvana Nicola Department of Agricultural, Forest and Food Sciences, DISAFA, Vegetable Crops \& Medicinal and Aromatic Plants, VEGMAP, University of Turin, Grugliasco (Turin), Italy

Carlo Nicoletto Department of Agronomy, Food, Natural Resources, Animal and Environment (DAFNAE), University of Padova, Padova, Italy

Fabio Francesco Nocito Department of Agricultural and Environmental Sciences Production, Landscape, Agroenergy, Università degli Studi di Milano, Milan, Italy

Monica Ozores-Hampton Institute of Food and Agricultural Sciences, Horticultural Sciences Department, University of Florida, Gainesville, FL, USA

Francisco M. Padilla Department of Agronomy, University of Almeria, Almeria, Spain

Giuseppe Pignata Department of Agricultural, Forest and Food Sciences, DISAFA, Vegetable Crops \& Medicinal and Aromatic Plants, VEGMAP, University of Turin, Grugliasco (Turin), Italy

Paolo Polinori Department of Economics, University of Perugia, Perugia, Italy

Miguel Quemada Department of Agricultural Production and CEIGRAM, Technical University of Madrid, Madrid, Spain

Gian Attilio Sacchi Department of Agricultural and Environmental Sciences Production, Landscape, Agroenergy, Università degli Studi di Milano, Milan, Italy

Paolo Sambo Department of Agronomy, Food, Natural Resources, Animal and Environment (DAFNAE), University of Padova, Padova, Italy

Eric H. Simonne Institute of Food and Agricultural Sciences, Horticultural Sciences Department, University of Florida, Gainesville, FL, USA

Francesco Tei Department of Agricultural, Food and Environmental Sciences, University of Perugia, Perugia, Italy

Rodney B. Thompson Department of Agronomy, University of Almeria, Almeria, Spain 
Giacomo Tosti Department of Agricultural, Food and Environmental Sciences, University of Perugia, Perugia, Italy

Nicolas Tremblay Agriculture and Agri-Food Canada, Saint-Jean-sur-Richelieu Research and Development Centre, QC, Canada

Luca Turchetti Council for the Research and the Analysis of the Agricultural Economy, Rome and Perugia, Italy 